\title{
Inhibitory action of Celastrol on hypoxia-mediated angiogenesis and metastasis via the HIF-1 $\alpha$ pathway
}

\author{
LILI HUANG ${ }^{1}$, ZEFU ZHANG ${ }^{2}$, SHENG ZHANG $^{1}$, JINGHUA REN ${ }^{1}$, \\ RUIGUANG ZHANG ${ }^{1}$, HUI ZENG ${ }^{1}$, QINGFENG LI $^{1}$ and GANG WU ${ }^{1}$ \\ ${ }^{1}$ Cancer Center, Union Hospital, Tongji Medical College, Huazhong University of Science and Technology, Wuhan; \\ ${ }^{2}$ Department of Radiology, The Fifth Affiliated Hospital of Sun Yat-sen University, Zhu Hai, P.R. China
}

Received October 15, 2010; Accepted December 2, 2010

DOI: $10.3892 / \mathrm{ijmm} .2011 .600$

\begin{abstract}
Celastrol, a natural biologically active compound isolated from Tripterygium wilfordii Hook F root extracts, has been shown to possess antitumor properties and therefore, is an interesting candidate for the development of novel chemotherapeutic cancer agents. In this study, we have demonstrated that Celastrol is a potent inhibitor of hypoxia-induced angiogenic and metastatic activity as shown by a decrease in the proliferation of both endothelial and cancer cells, blocking of migration as well as of tube formation of endothelial cells, and by inhibition of cancer cell invasion under hypoxic conditions. Moreover, Celastrol decreased hypoxia-inducible factor- $1 \alpha$ (HIF-1 $\alpha$ ) mRNA levels under both normoxia and hypoxia and inhibited hypoxia-induced accumulation of nuclear HIF-1 $\alpha$ protein. Meanwhile, inhibition of nuclear HIF-1 $\alpha$ protein levels were accompanied by a reduction in the transcriptional activity of HIF-1 $\alpha$ target genes, including VEGF. In addition, the inhibitory effect of Celastrol on HIF-1 $\alpha$ protein was partly due to its suppression of HSP90 activity. We conclude that Celastrol regulates HIF-1 $\alpha$ at multiple levels that may together or individually contribute to its antitumor activity against hypoxia-induced angiogenesis and metastasis.
\end{abstract}

\section{Introduction}

Intratumoral hypoxia $\left(0.05-5 \% \mathrm{O}_{2}\right)$ is a common pathophysiologic feature of solid tumors, from the smallest tumor of a few millimeters in diameter to the largest tumor (1). The hypoxic tumor environment results in aggressive and metastatic cancer phenotypes that are associated with resistance to radiation therapy, chemotherapy, and a poor treatment outcome $(2,3)$. Hypoxia-inducible factor-1 (HIF-1) is the key

Correspondence to: Dr Gang Wu, Cancer Center, Union Hospital, Tongji Medical College, Huazhong University of Science and Technology, Wuhan 430024, P.R. China

E-mail: wugangzr2001@yahoo.com.cn

Key words: Celastrol, hypoxia-inducible factor-1 $\alpha$, angiogenesis, metastasis factor in this process, regulating transcription of more than 70 hypoxia-activated genes, including glucose transporter-1 (Glut-1), vascular endothelial growth factor (VEGF), VEGF receptor-1 (Flt-1), and carbonic anhydrase 9 (CA9) that range in function from those that promote anaerobic metabolism to those that initiate tumor angiogenesis and metastasis (4). HIF-1 is a heterodimeric transcription factor composed of HIF- $1 \alpha$ and constitutively expressed HIF- $1 \beta$ subunits. Its biological activity depends on the amount of HIF-1 $\alpha$, which is tightly regulated by oxygen tension (5). Under hypoxic conditions, the blockage of prolyl hydroxylation, ubiquitination and degradation lead to the remarkable accumulation and translocation of HIF-1 $\alpha$ protein to the nucleus, where it forms an active complex with HIF-1 $\beta$. Overexpression of HIF-1 $\alpha$ has been demonstrated in human cancers as compared with the respective normal tissues, making it a potential therapeutic target in oncologic drug discovery (6). Inhibition of HIF-1 $\alpha$ or its genetic disruption can not only block tumor cell growth, but also inhibit tumor angiogenesis and metastasis, so that these tumor cells would be unable to respond to the hypoxic stimulus and to progress $(7,8)$.

Celastrol, a quinone methide triterpene, which is a pharmacologically active compound present in Tripterygium wilfordii Hook F root extracts, has been widely used to treat autoimmune diseases, chronic inflammation, neurodegenerative diseases, and many types of cancer (9). Recently, Celastrol was shown to interrupt the molecular chaperone heat shock protein 90 (HSP90) via preventing the interaction of HSP90 and $\mathrm{Cdc} 37$ protein, a co-chaperone in the HSP37-Cdc37 complex mediating the binding of protein kinases to HSP90 (10). As a molecular chaperone, HSP90 contributes to the stability and folding of key proteins involved in the hallmark traits of cancer. It has been proven that HSP90 interacts with HIF-1 $\alpha$ and mediates the proper folding and hypoxic stabilization of HIF-1 $\alpha$ (11-13). High-dose treatment of HeLa cells with three different HSP90 inhibitors reduced hypoxiamediated HIF-1 $\alpha$ activity and protein levels (14). In light of these, we hypothesize that pharmacological inactivation of HSP90 by Celastrol may affect the HIF-1 $\alpha$ pathway and impair hypoxia-stimulated angiogenesis and metastasis. To test this hypothesis, we first identified the anticancer efficacy of Celastrol in both human endothelial (EA.hy926) and cancer cells (HepG2 and A549) under a hypoxic environment. Then, 
we evaluated the inhibitory effect of Celastrol on hypoxiainduced angiogenesis by endothelial cells and invasiveness of cancer cells. Furthermore, we investigated the reduction of the mRNA levels and expression of nuclear HIF-1 $\alpha$ protein by Celastrol. Meanwhile, the decrease in HIF-1 $\alpha$ expression was associated with dramatic down-regulation of HIF-1 $\alpha$ transcriptional activity and of the mRNA expression of the downstream targeted genes. Finally, our results supported that Celastrol exerted its repressive effect on the HIF-1 $\alpha$ pathway, at least in part, via restraining HSP90 activity. These findings provide further evidence that Celastrol can be a promising chemopreventive and anticancer agent in human cancer by inhibiting tumor angiogenesis and metastasis via targeting the well-known tumor survival factor, HIF-1 $\alpha$, under hypoxic stress.

\section{Materials and methods}

Reagents and antibodies. Celastrol (Cayman Chemicals) was solubilized in $100 \%$ DMSO at $100 \mu \mathrm{g} / \mathrm{ml}$ concentration and frozen at $-20^{\circ} \mathrm{C}$ in small aliquots until needed. The antibody against $\beta$-actin was obtained from Santa Cruz Biotechnology. The antibody, anti-HIF-1 $\alpha$ was purchased from BD Biosciences. The antibodies anti-HSP90, anti-Akt, phosphorylated-specific anti-Akt (Ser473), anti-Met, phosphorylated-specific anti-Met (Tyr1234/1235) were purchased from Cell Signaling Technology.

Cell lines and hypoxic treatment. Human umbilical vein vascular endothelial cells (EA.hy926) and human hepatocellular liver carcinoma cells (HepG2) were cultured in DMEM. Human lung adenocarcinoma epithelial cells (A549) were maintained in RPMI-1640. All media were supplemented with $10 \% \mathrm{FBS}$ and antibiotics. All cells were cultured at $37^{\circ} \mathrm{C}$ in a humidified atmosphere and $5 \% \mathrm{CO}_{2}$ in air. For hypoxic exposure, the cells were placed in a sealed modular incubator chamber flushed with $1 \% \mathrm{O}_{2}, 5 \% \mathrm{CO}_{2}$, and $94 \% \mathrm{~N}_{2}$.

Cell proliferation assay. One day before the assay, cells were seeded at $10^{5}$ cells/well in a 96-well plates in a volume of $200 \mu \mathrm{l}$. The next day, the cells were treated with increasing concentrations of Celastrol and cultured under hypoxic conditions for 6 or $16 \mathrm{~h}$. All samples, including controls, contained $0.1 \%$ DMSO. Viable cells were determined using methylthiazol-tetrazolium (MTT). The absorbance at $562 \mathrm{~nm}$ was determined using the ELx 808 microplate reader (Bio-Tek, Winooski, VT). Five wells were assayed at each concentration and the mean absorbance was determined. The inhibition rate was calculated by the formula: inhibition rate of proliferation $(\%)=\left(\mathrm{A}_{\text {control }}-\mathrm{A}_{\text {Celastrol }}\right) / \mathrm{A}_{\text {control }} \times 100 \%$. The data were expressed as mean $\pm \mathrm{SD}$ from three independent experiments.

Cell migration and invasion assay. The migration and invasion assays were performed in a transwell Boyden Chamber (Costar) using a polycarbonate filter with an $8 \mu \mathrm{m}$ pore size in a 24-well plate. For cell invasion assay, the filter membranes were coated with $30 \mu \mathrm{g}$ matrigel (BD Biosciences). Confluent cells (EA.hy926 cells for the migration assay; HepG2 and A549 cells for invasion assay) were added to the inner chamber of the insert in $200 \mu \mathrm{l}$ of serum-free medium and $500 \mu \mathrm{l}$ of medium with $10 \%$ FBS were added to the lower chamber. To determine the effect of Celastrol on cell migration and invasion, indicated concentrations of Celastrol were added to the lower chamber and $0.1 \%$ DMSO was used as a control. After incubation under normoxia or hypoxia for $16 \mathrm{~h}$ at $37^{\circ} \mathrm{C}$, the cells remaining on the upper surface were gently removed using a cotton-tipped swab. The cells on the opposite surface of the filter membrane were stained with $0.1 \%$ crystal violet and counts were obtained from five randomly selected fields (x400 magnification) using a computer imaging system.

Tube formation assay. Matrigel (200 $\mu 1$ of $10 \mathrm{mg} / \mathrm{ml})$ was pipetted onto 24-well culture plates and polymerized for $30 \mathrm{~min}$ at $37^{\circ} \mathrm{C}$. EA.hy 926 cells $\left(1 \times 10^{5}\right)$ were seeded on the surface of the matrigel and cultured in complete medium containing various concentrations of Celastrol or $0.1 \%$ DMSO as a control under hypoxic conditions for $16 \mathrm{~h}$. Morphological change of the cells were observed under a microscope and five randomly chosen fields were photographed at x200 magnification using the ImagePro Plus software (Media Cybernetics, Silver Spring, MD).

HIF-1 $\alpha$ immunofluorescence. Exponentially growing cells were plated on $12-\mathrm{mm}$ glass coverslips into 24 -well plates and cells were allowed to attach overnight. The following day, cells were treated with the indicated drugs for $16 \mathrm{~h}$ under normoxia or hypoxia. Cells were fixed with methanol for $5 \mathrm{~min}$. The non-specific binding sites were blocked with 4\% BSA in PBS for 30 min. The cells were incubated with a mouse monoclonal anti-HIF- $1 \alpha \operatorname{IgG}$ antibody overnight at $4^{\circ} \mathrm{C}$ diluted 1:200 in 1\% BSA in PBS followed by a Cy3-coupled secondary anti-mouse antibody diluted 1:500 with $1 \%$ BSA in PBS. Subsequently, all cells were stained with Hoechst 33258 dye for $2 \mathrm{~min}$. After extensive washings with PBS, the coverslips were mounted onto the glass slides and the cells were examined with fluorescence microscopy (Axioplan 2000, Carl Zeiss Vision, Mannheim, Germany).

Real-time PCR. Total cellular RNA was extracted and purified using the TRIzol reagent (Invitrogen). Total RNA $(1 \mu \mathrm{g})$ was used to transcribe the first-strand cDNA with SuperScript II reverse transcriptase (Invitrogen). The realtime PCR was completed on the ABI PRISM Sequence Detector 7000 (PerkinElmer, Branchburg, NJ). SYBR-Green PCR Master mix was purchased from Applied Biosystems. Initial template concentrations of each sample were calculated by comparison with serial dilutions of a calibrated standard. The relative abundance of sample transcripts was quantified using the comparative $\mathrm{Ct}$ method with $18 \mathrm{~s}$ as an internal control. Primers were as follows: human HIF-1 $\alpha$ forward, 5'-GGCGCGAACGACAAGAAAAAG-3', hHIF-1 $\alpha$ reverse, 5'-CCTTATCAAGATGCGAACTCACA-3'; human VEGF forward, 5'-CAACATCACCATGCAGATTATGC-3', hVEGF reverse, 5'-GCTTTCGTTTTTGCCCCTTTC-3'; human CA9 forward, 5'-GGGTGTCATCTGGACTGTGTT-3', hCA9 reverse, 5'-CTTCTGTGCTGCCTTCTCATC-3'; human 18s forward, 5'-TTGACGGAAGGGCACCACCAG-3', h18s reverse 5'-GCACCACCACCCACGGAATC G-3'. Using $\mathrm{Ct}$ values (cycle number in which the sample crosses the threshold value) for samples (sam) and controls, the $\Delta \mathrm{Ct}$ was 
calculated: $\Delta \mathrm{Ct}=\mathrm{Ct}_{\mathrm{sam}}-\mathrm{Ct}_{18 \mathrm{~s}}$. The values for each sample were then compared with the control sample (ctl): $\Delta \Delta \mathrm{Ct}=$ $\Delta \mathrm{Ct}_{\mathrm{sam}}-\Delta \mathrm{Ct}_{\mathrm{ctl}}$. The fold change of the mRNA level to the control was $2^{-\Delta \Delta C t}$.

ELISA. Secreted VEGF from treated cells was measured using an ELISA kit (R\&D Systems) for VEGF protein according to the manufacturer's instructions. Absorbance was determined by a SpectraFluor Plus plate reader (Tecan) at $450 \mathrm{~nm}$. The results were expressed as the concentration of VEGF $(\mathrm{pg} / \mathrm{ml})$ per the total protein amount from each well.

Western blot analysis. Treated and untreated cells were lysed with buffer containing $50 \mathrm{mM}$ Tris- $\mathrm{HCl}(\mathrm{pH} 7.5), 5 \mathrm{mM}$ EDTA, $150 \mathrm{mM} \mathrm{NaCl}, 0.5 \%$ Triton $\mathrm{X}-100,10 \mathrm{mM}$ sodium fluoride, $20 \mathrm{mM} \beta$-mercaptoethanol, $250 \mu \mathrm{M}$ sodium orthovanadate, $1 \mathrm{mM}$ phenylmethylsulfonyl fluoride, and complete protease inhibitor cocktail (Sigma), and were incubated at $4^{\circ} \mathrm{C}$ for $30 \mathrm{~min}$. The lysates were ultrasonicated and centrifuged at $14,000 \mathrm{x} \mathrm{g}$ for $15 \mathrm{~min}$. The proteins were separated on a 8-10\% gradient SDS-PAGE and transferred onto nitrocellulose membranes (Hybond ECL, Amersham Pharmacia, Piscataway, $\mathrm{NJ})$. After blocking with skimmed milk in 5\% TBST, the membranes were incubated overnight at $4^{\circ} \mathrm{C}$ with primary antibodies against HIF-1 $\alpha$, HSP90, Akt, phosphorylated Akt (Ser473), Met, or phosphorylated Met (Tyr1234/1235). After washing three times with TBST for $5 \mathrm{~min}$, the membranes were incubated with secondary anti-rabbit or anti-mouse antibodies for $1 \mathrm{~h}$ at room temperature and were then developed by ECL.

Statistical analysis. Data are shown as the means \pm SD. Multiple group comparison was performed by one-way ANOVA for comparison of means. Comparisons between 2 groups were analyzed by the unpaired Student's t-test. P-values of $<0.05$ were considered statistically significant.

\section{Results}

Celastrol inhibited the proliferation of cancer and endothelial cells under hypoxia. Uncontrolled proliferation is one of the most distinguishing traits of cancer cells. To determine direct cytotoxic effect of Celastrol on cancer and endothelial cells under hypoxic conditions, we performed cell death assays in HepG2, A549 and EA.hy926 cells treated with Celastrol (Fig. 1). Although Celastrol treatment at a concentration of $4 \mu \mathrm{g} / \mathrm{ml}$ for $6 \mathrm{~h}$ led to a $17.44 \%$ and a $14.93 \%$ inhibiton on HepG2 and A549 cells, respectively, it displayed dramatic cytotoxicity with an $\mathrm{IC}_{50}$ of 6.54 and $4.32 \mu \mathrm{g} / \mathrm{ml}$ when the duration of treatment was lengthened to $16 \mathrm{~h}$. Similar antiproliferative effects of Celastrol were also observed in endothelial cells. Our findings suggest that Celastrol could inhibit the proliferation of both cancer and endothelial cells in a dose- and time-dependent manner under hypoxia. In the following studies, all of the functional assays were performed using optimal sub-cytotoxic concentrations of Celastrol.

Celastrol reduced the hypoxia-induced EA.hy926 migration. Endothelial cell migration is a defining characteristic of tumor angiogenesis. Hypoxia is a potent stimulator of the migration
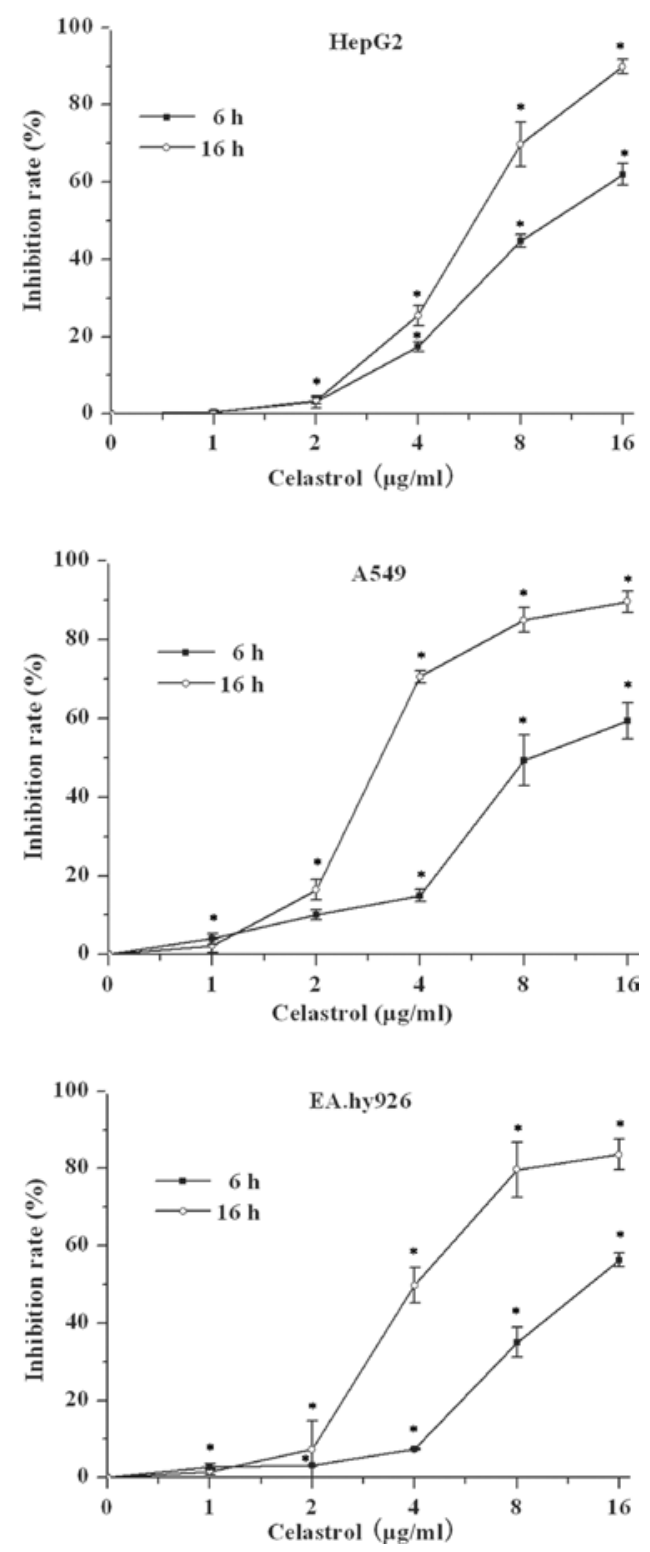

Figure 1. Celastrol inhibited the proliferation of tumor and endothelial cells under hypoxia. HepG2, A549 and EA.hy926 cells were treated with the indicated concentrations of Celastrol under hypoxic conditions. All concentrations included $0.1 \%$ DMSO as the control (concentration $0 \mu \mathrm{g} / \mathrm{ml}$ ). After $6 \mathrm{~h}$ or $16 \mathrm{~h}$, the cells were processed for the MTT proliferation assay and growth inhibition was measured as described in Materials and methods. Points, inhibition ( $\mathrm{n}=3$ ); bars, SD. ${ }^{*} \mathrm{P}<0.05$, vs. control.

of endothelial cells. To determine the effect of Celastrol on hypoxia-induced EA.hy926 migration, we cultured EA.hy926 cells under both normoxic and hypoxic conditions for $16 \mathrm{~h}$. As shown in Fig. 2A, the number of EA.hy926 cells that had migrated to the lower side of the filter in the Boyden Chamber was increased under hypoxic conditons as compared to that under normoxic conditions. Celastrol treatment at the concentrations of 0.75 to $2 \mu \mathrm{g} / \mathrm{ml}$ remarkably reduced the number of migrated cells in the hypoxic conditions, with an $\mathrm{IC}_{50}$ value of $1.35 \mu \mathrm{g} / \mathrm{ml}$, indicating that Celastrol suppressed the stimulatory effect of hypoxia-activated migration of endothelial cells (Fig. 2C). No cytotoxicity against EA.hy926 cells was observed under the same concentrations of Celastrol for $16 \mathrm{~h}$ used in the above experiment, indicating the inhibition of migration was not due to the cytotoxic effect of Celastrol. 

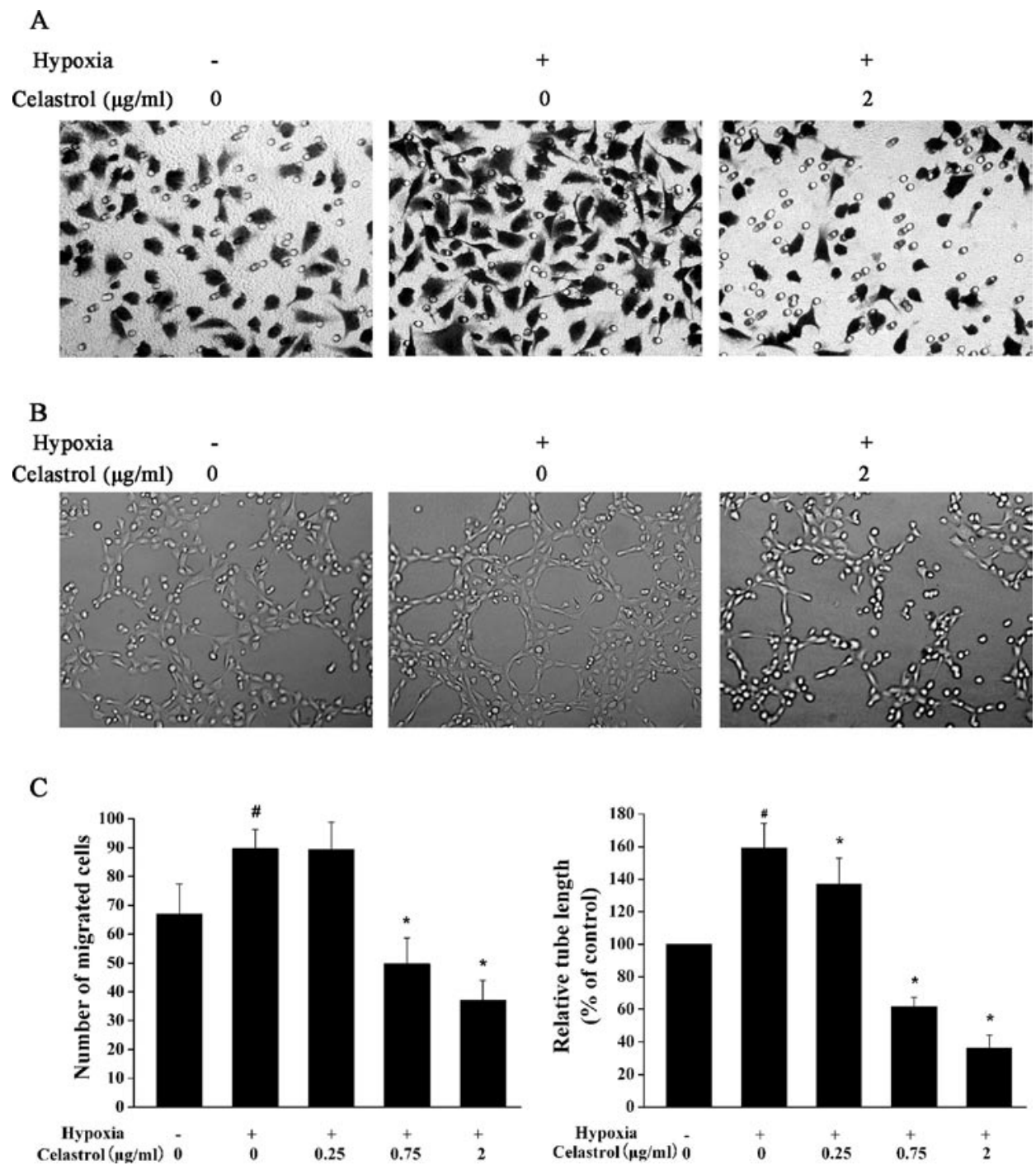

Figure 2. Celastrol inhibits hypoxia-induced angiogenesis. Celastrol dose-dependently inhibits the hypoxia-induced migration and tube formation of EA.hy926 cells (A) Images of migrated EA.hy926 cells in the absence or presence of Celastrol under hypoxia for $16 \mathrm{~h}$ (x400 magnification). (B) Images of tube formation by EA.hy926 cells in the absence or presence of Celastrol under hypoxia for $16 \mathrm{~h}$. (C) Left panel, quantitation of migrated cells. The migrated cells were counted in five randomly selected microscopic fields (x400 magnification). Right panel, quantification of the length of the tube network was obtained using the Image-Pro Plus software. Cells were counted from five randomly selected microscopic fields (x200 magnification). The relative tube length was obtained by comparing the length of the tubes formed in the presence of Celastrol under hypoxia with that formed under normoxia. Values represent the means $\pm \mathrm{SD}, \mathrm{n}=3$. ${ }^{*} \mathrm{P}<0.05$ vs. normoxia. ${ }^{*} \mathrm{P}<0.05$ vs. hypoxia only.

Celastrol impaired hypoxia-induced tube formation of EA.hy926. In the latter stages of angiogenesis, endothelial cells self-assemble into tubes to form new blood vessels. We therefore, evaluated the effect of Celastrol on the formation of functional tubes by hypoxic EA.hy926 cells plated on the matrigel-coated plates. Under normoxic conditions, EA.hy926 cells were scattered throughout the plate. After exposure to hypoxia for $16 \mathrm{~h}$, EA.hy926 cells formed a complete network of tube-like structures, indicating that hypoxia exhibited a significantly enhanced angiogenesis stimulating activity. Tubular formation by hypoxic EA.hy 926 was impaired by Celastrol in a concentration-dependent manner for $16 \mathrm{~h}$ (Fig. 2B and C). No obvious changes in cell viability were observed under this concentration range of Celastrol. Therefore, Celastrol was shown to interfere with the ability of EA.hy926 cells to form vessel-like tubes under hypoxia and this effect was not ascribed to non-specific tumor cell toxicity.
Celastrol prevented hypoxia-induced invasion of cancer cells. Hypoxia represents a physiological stimulus for tumor cell invasion and metastasis. The progression of tumors to an invasive phenotype and ultimately the formation of metastasis is associated with increased mortality (15). We examined whether hypoxic conditions enhanced the invasiveness of cancer cells and whether Celastrol could prevent the invasion ability of cancer cells by the matrigel-coated Boyden chamber transwell assay. As shown in Fig. 3A and C, an increase in the baseline invasiveness of cancer cells was observed under hypoxic conditions as compared to normoxic conditions. The number of HepG2 cells that invaded through the matrigel membrane increased from $55.67 \pm 3.06$ per field under normoxia to $136.67 \pm 6.11$ per field under hypoxia. The stimulatory effect of hypoxia on the invasiveness of cancer cells was reduced by Celastrol in a dose-dependent manner. The number of hypoxic invaded cells decreased from $136.67 \pm 6.11$ per field to $46 \pm 4$ per field with the addition of $2 \mu \mathrm{g} / \mathrm{ml}$ Celastrol. Similar 

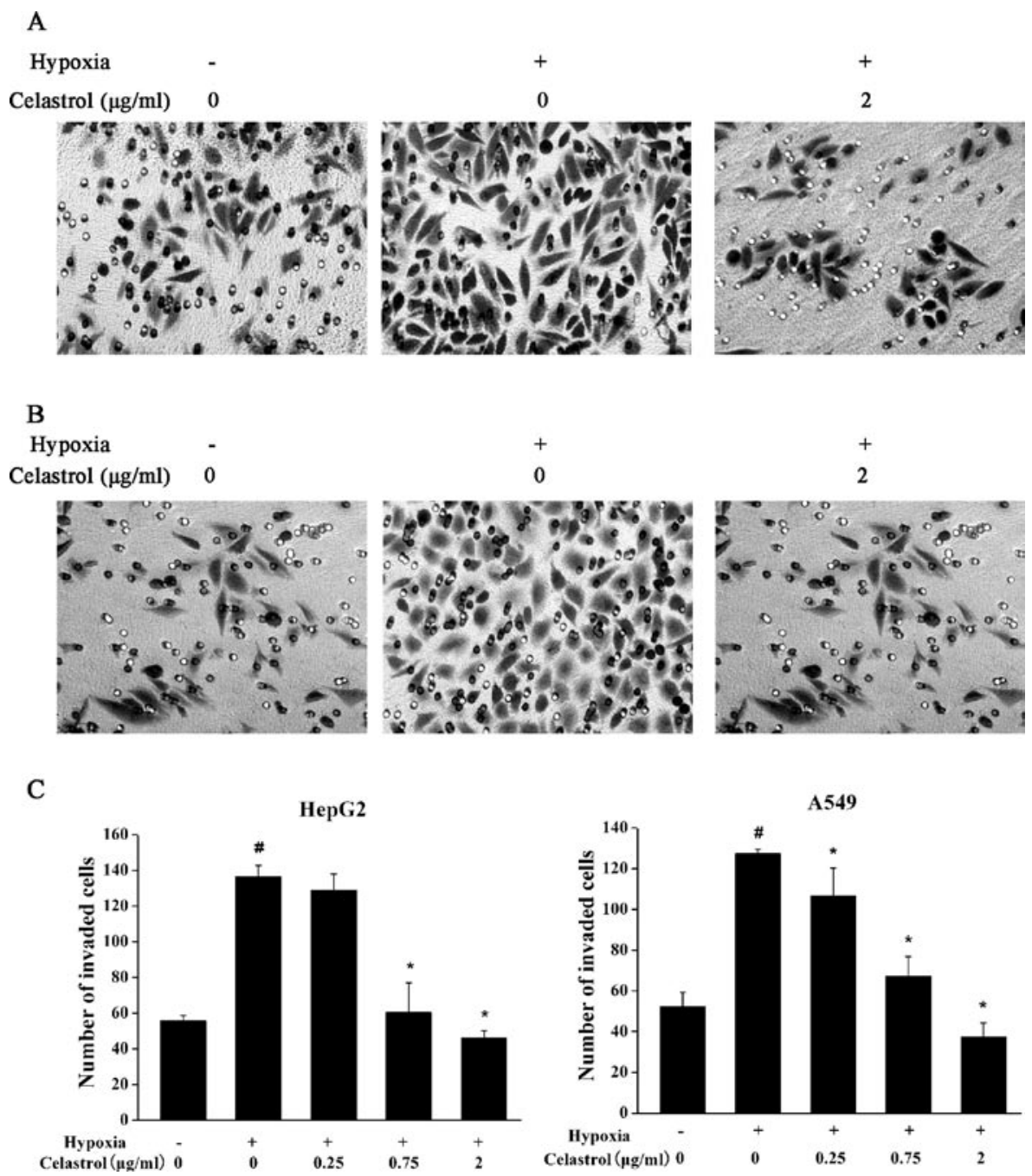

Figure 3. Celastrol inhibits hypoxia-invasion of cancer cells. Celastrol dose-dependently inhibited the invasion of HepG2 and A549 cancer cells. (A) Images of invaded HepG2 (A) and invaded A549 (B) cells (x400 magnification). (C) The HepG2 and A549 cells that had invaded to the lower chamber were counted in five randomly selected microscopic fields ( $\mathrm{x} 400$ magnification). Values represent means $\pm \mathrm{SD}, \mathrm{n}=3 .{ }^{*} \mathrm{P}<0.05$ versus normoxia. ${ }^{*} \mathrm{P}<0.05$ vs. hypoxia only.

results were obtained from A549 cells under the same conditions (Fig. 3B and C). These results indicate that Celastrol could suppress the hypoxia-stimulated invasion ability of cancer cells. The inhibitory effect of Celastrol on the invasion of cancer cells was not due to the cytotoxic effect of Celastrol, because the viability of those cancer cells was barely affected by Celastrol at the concentrations used in this experiment.

Celastrol down-regulated HIF-1 $\alpha$ nuclear protein expression in HepG2 cells. Hypoxia-induced angiogenesis and metastasis are mainly mediated by VEGF via activation of the HIF-1 $\alpha$ signaling pathway (16). In this experiment, we sought to investigate whether Celastrol could regulate HIF-1 $\alpha$ protein expression, which might contribute to the anti-angiogenic effect of Celastrol. First, immunofluorescent staining illustrated that under normoxic conditions, HIF-1 $\alpha$ immunoreactivity of rather low intensity was preferentially localized in the cytoplasmic compartment of the cells, as assessed by using monoclonal anti-HIF-1 $\alpha$ antibody. Exposure of HepG2 cells to hypoxic conditions for $16 \mathrm{~h}$ resulted in an almost complete nuclear translocation of HIF-1 $\alpha$ with minimal detectable immunoreactivity remaining in the cytoplasm. Cells were subsequently treated with 0.25 or $2 \mu \mathrm{g} / \mathrm{ml}$ Celastrol for $16 \mathrm{~h}$ under hypoxic conditions. Addition of $0.25 \mu \mathrm{g} / \mathrm{ml}$ Celastrol did not affect the expression and nuclear translocation of HIF-1 $\alpha$, whereas cells treated with $2 \mu \mathrm{g} / \mathrm{ml}$ Celastrol showed cytoplasmic staining and minimal nuclear staining (Fig. 4A). Thus, Celastrol treatment notably blocked the hypoxiainduced accumulation of nuclear HIF-1 $\alpha$, which was further illustrated by Western blot (Fig. 4B). Using cell fractionation procedures, we found that the basal levels of nuclear HIF-1 $\alpha$ protein were low to undetectable in HepG2 cells under normal conditions. Exposure of HepG2 cells to $1 \%$ oxygen for $16 \mathrm{~h}$ resulted in hypoxic stabilization of nuclear HIF-1 $\alpha$. The protein levels of nuclear HIF-1 $\alpha$ began to decrease at $2 \mu \mathrm{g} / \mathrm{ml}$ of Celastrol and were dramaticly suppressed at $4 \mu \mathrm{g} / \mathrm{ml}$. The results suggest that Celastrol might down-regulate the protein levels of nuclear HIF-1 $\alpha$ via affecting its translocation.

Celastrol inhibited HIF-1 $\alpha$ transcription. To further investigate the effect of Celastrol treatment on HIF-1 $\alpha$ transcription, we treated HepG2 and A549 cells with increasing concentrations of Celastrol under normoxic or hypoxic conditions, and HIF-1 $\alpha$ mRNA levels were tested by real-time PCR. HIF-1 $\alpha$ 

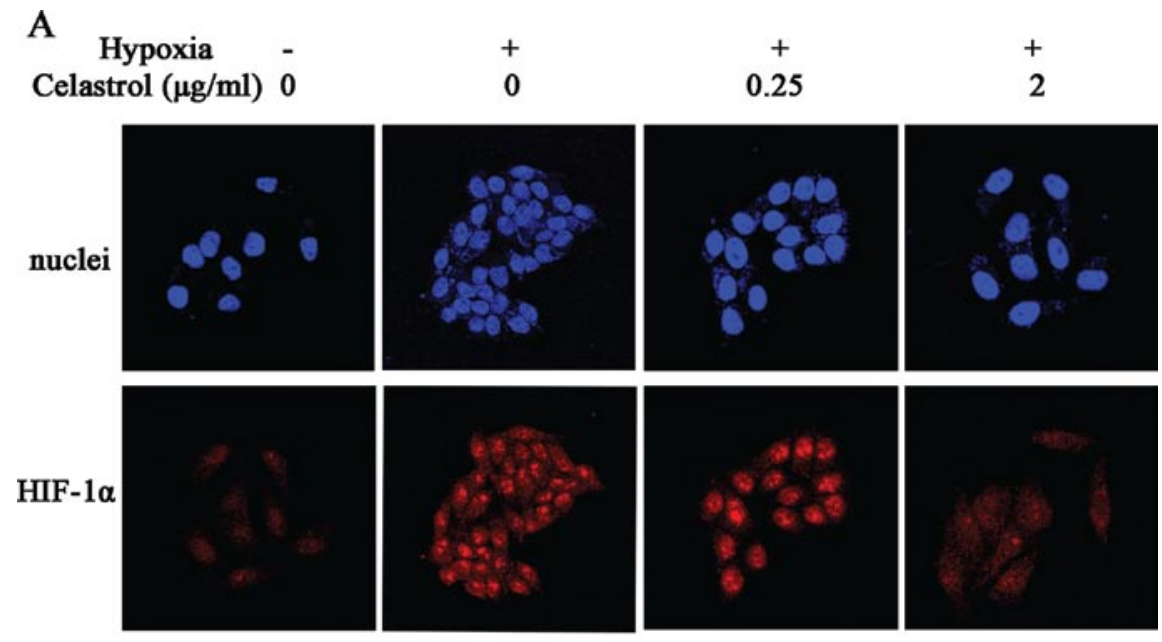

B

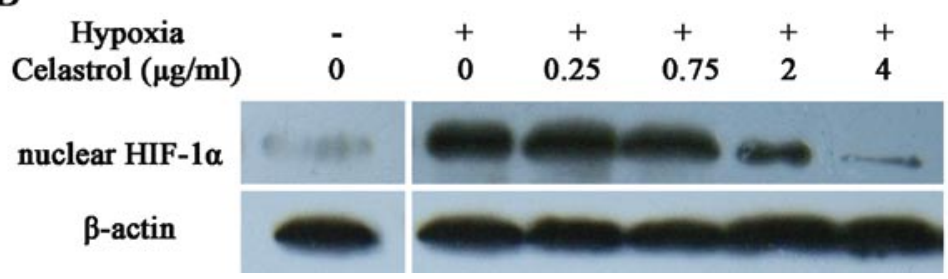

Figure 4. Celastrol inhibits nuclear HIF-1 $\alpha$ protein expression. (A) Celastrol affected HIF-1 $\alpha$ protein translocation. HepG2 cells were exposed to normoxic or hypoxic conditions without or with the addition of 0.25 or $2 \mu \mathrm{g} / \mathrm{ml}$ Celastrol for $16 \mathrm{~h}$. The cells were prepared for indirect immunofluorescence analysis as described in Materials and methods. Upper panel, Hoechst 33258 staining of the nuclei. Lower panel, anti-HIF-1 $\alpha$ staining using a Cy3-conjugated secondary antibody. (B) Celastrol inhibits nuclear HIF-1 $\alpha$ protein levels. HepG2 cells were treated for $16 \mathrm{~h}$ with Celastrol at the indicated concentrations under hypoxic conditions. Subsequently, nuclear protein was extracted, and HIF-1 $\alpha$ was detected by immunoblotting.

mRNA levels were significantly inhibited by a 16-h Celastrol treatment even of a very low concentration $(0.75 \mu \mathrm{g} / \mathrm{ml})$ in HepG2 cells under both normoxia and hypoxia. Treatment with $4 \mu \mathrm{g} / \mathrm{ml}$ Celastrol reduced HIF- $1 \alpha$ mRNA by $\sim 85 \%$ under both normoxia and hypoxia (Fig. 5A). Similar results were obtained by treating A549 cells with Celastrol (data not shown). Hence, we showed that Celastrol treatment decreased HIF-1 $\alpha$ mRNA levels in a dose-dependent manner under both normoxia and hypoxia.

Celastrol inhibited HIF-1 $\alpha$ transcriptional activity. The effectiveness of the hypoxia response depends on the transactivation of HIF-1 target genes. VEGF, an immediate downstream target gene of HIF-1 $\alpha$, plays a pivotal role in tumor angiogenesis, especially under conditions of intratumoral hypoxia. To determine whether Celastrol could down-regulate hypoxiamediated VEGF expression, we examined VEGF mRNA and protein levels in HepG2 and A549 cells by real-time PCR and an ELISA assay. As expected, hypoxic exposure of the untreated cells resulted in a $>10$-fold induction in VEGF mRNA in HepG2 cells. Consistent with the decreased levels in HIF-1 $\alpha$ protein by Celastrol, VEGF mRNA levels were inhibited by Celastrol in a dose-dependent manner (Fig. 5B). Similar results were obtained following treatment of A549 cells with Celastrol (data not shown). VEGF mRNA downregulation by Celastrol was paralleled by a reduced protein secretion. A 3.15-fold up-regulation of secreted VEGF was observed after $16 \mathrm{~h}$ culture under hypoxia. Treatment with $0.75 \mu \mathrm{g} / \mathrm{ml}$ Celastrol inhibited hypoxia-induced VEGF protein by $70 \%$ (Fig. 5B). These results demonstrate that Celastrol is a potent inhibitor of VEGF production induced by hypoxia.

To further examine the effect of Celastrol on the transcriptional activity of HIF-1 $\alpha$, the mRNA levels of carbonic anhydrase 9 (CA9) were examined. Among 50 genes targeted by HIF, CA9 is one of the most uniformly induced genes. Expression of CA9 in many common epithelial cancers can be used as a marker of hypoxia associated with poor prognosis $(17,18)$. Exposure of HepG2 and A549 cells for $16 \mathrm{~h}$ under hypoxia indeed resulted in a strong increase in CA9 mRNA levels compared with cells grown under normoxia. Treatment of HepG2 and A549 cells with Celastrol resulted in a decrease of hypoxia-induced CA9 mRNA expression to different extents (60-90\% and 20-90\%, respectively; Fig. 5C). Hence, this result further confirms that Celastrol inhibits hypoxiamediated transcriptional activation of HIF-1 $\alpha$ target genes.

Celastrol decreased HSP90 client protein levels. HIF-1 $\alpha$ is associated with HSP90 and binding of HIF-1 $\alpha$ to HSP90 prevents HIF-1 $\alpha$ degradation. Previous studies have found that Celastrol disrupted the associaton of HSP90 with co-chaperones p23 and Cdc37 and affected the binding of protein kinases to HSP90 (10). To determine whether Celastrol affects hypoxia-induced HIF-1 $\alpha$ expression via disturbing HSP90 function, we examined the protein levels of HSP90 and other HSP90 clients in HepG2 cells by Western blot analysis. Very small or no effect of Celastrol treatment on the levels of HSP90 protein was observed after $16 \mathrm{~h}$ under hypoxic condition. The result was consistant with previous findings 
A
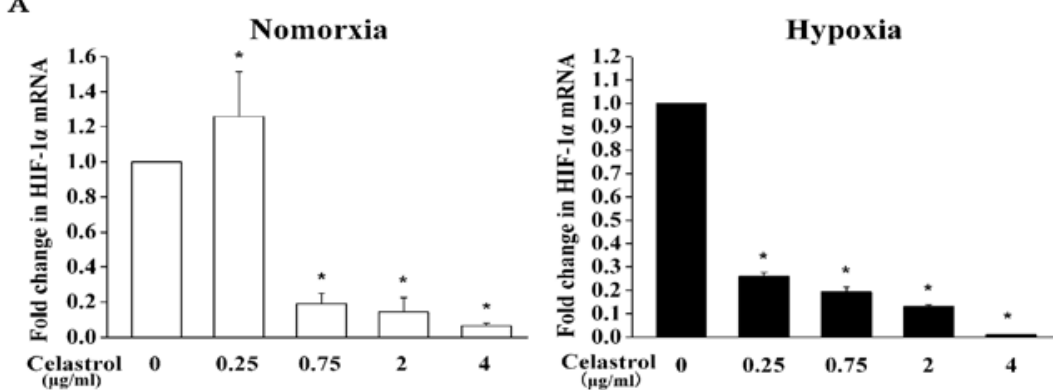

B
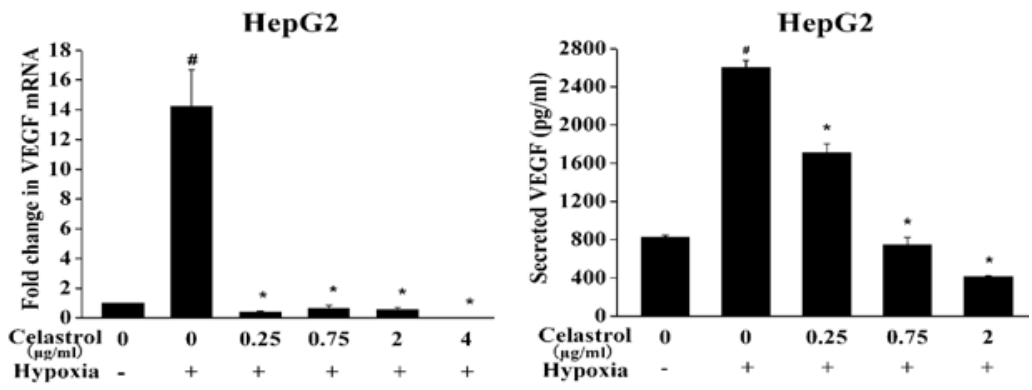

C
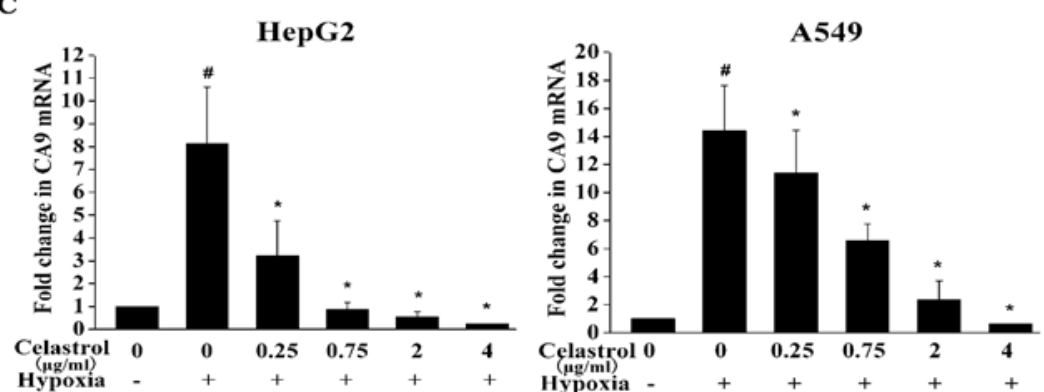

Figure 5. Celastrol inhibits HIF-1 $\alpha$ transcription and transcriptional activity. (A) Celastrol decreased HIF-1 $\alpha$ mRNA levels. Total RNA was isolated from HepG2 cells treated with indicated Celastrol concentrations under normoxic and hypoxic conditions for $16 \mathrm{~h}$. HIF-1 $\alpha$ transcript levels were analyzed by real-time PCR using primers specific to HIF- $1 \alpha$ and normalized to levels of $18 \mathrm{~s}$. Values represent means $\pm \mathrm{SD}, \mathrm{n}=3$. ${ }^{*} \mathrm{P}<0.05$ vs. hypoxia only. (B), Celastrol inhibits VEGF mRNA and protein levels. HepG2 cells were exposed to normoxic or hypoxic conditions without or with the addition of Celastrol for $16 \mathrm{~h}$ Left panel, the cells were lysed; total RNA was extracted and reverse transcribed; and VEGF transcript levels were analyzed by real-time PCR using primers specific to VEGF and normalized to levels of 18s. Right panel, conditioned medium was harvested and assayed for the content of secreted VEGF by ELISA Values represent means $\pm \mathrm{SD}, \mathrm{n}=3 .{ }^{*} \mathrm{P}<0.05$ vs. normoxia. ${ }^{\mathrm{P}}<0.05$ vs. hypoxia only. (C) Celastrol inhibited CA9 mRNA levels. HepG2 and A549 cells were exposed to normoxic or hypoxic conditions without or with the addition of Celastrol for $16 \mathrm{~h}$. The cells were lysed; total RNA was extracted and reverse transcribed; and CA9 transcript levels were analyzed by real-time PCR. Values represent the means $\pm \mathrm{SD}, \mathrm{n}=3 .{ }^{*} \mathrm{P}<0.05$ vs. normoxia. ${ }^{*} \mathrm{P}<0.05$ vs. hypoxia only.

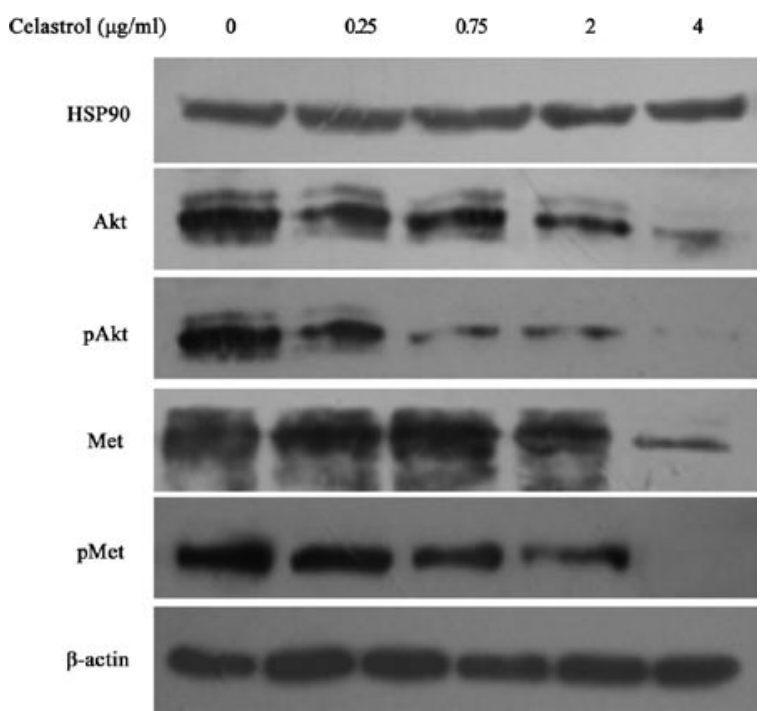

Figure 6. Celastrol decreased HSP90 client protein levels. HepG2 cells were treated for $16 \mathrm{~h}$ with Celastrol at the indicated concentrations under hypoxic conditions. Subsequently, cellular protein was extracted, and HSP90, Akt, pAkt, Met, pMet, and $\beta$-actin were detected by immunoblotting. reported by Zhang et al (10). Furthermore, the protein levels of Akt and Met, two well-known HSP90 clients, were measured. Treatment with Celastrol gradually down-regulated both Akt and its phosphorylated form in a dose-dependent manner under hypoxic conditions. A 16-h treatment of HepG2 cells with $0.25 \mu \mathrm{g} / \mathrm{ml}$ Celastrol had little effect on Akt and pAkt levels, but 2 and $4 \mu \mathrm{g} / \mathrm{ml}$ Celastrol was able to substantially decrease Akt and pAkt. Celastrol also decreased the protein levels of Met and abrogated its phosphorylated form at $4 \mu \mathrm{g} / \mathrm{ml}$. These results suggest that Celastrol suppresses HSP90 activity without changing the levels of HSP90 protein, which might contribute to a reduction of HIF-1 $\alpha$ nuclear protein expression.

\section{Discussion}

The utility of anti-angiogenic strategies for cancer control is strongly compromised by hypoxia-driven phenotypic changes, which make cancer cells more invasive and more prone to give rise to metastasis (19). Thus, it is evident that concurrent measures for blunting hypoxia-induced phenotypic 
changes will be required if antiangiogenic therapy is ever to achieve its goal of providing long-term cancer control and improved survival. Although traditional medicines are often viewed with skepticism by the Western medical establishment, they are a rich source of therapeutic strategies for the pharmaceutical industry and continue to provide front-line pharmacotherapy for many millions of people worldwide. Celastrol, a pharmacologically active compound present in Tripterygium wilfordii Hook F root extracts has been used traditionally for the treatment of arthritis and other diseases (9). Also, previous studies have shown that Celastrol has antitumor and antiangiogenic effect in several tumor cells, and the underlying mechanisms seem to be associated with their inhibitory effect on NF- $\kappa \mathrm{B}$ via TAK1 and $\mathrm{I} \kappa \mathrm{B} \alpha$ kinase, the proteasome, topoisomerase II, the AKT/mTOR/p70S6K pathway, VEGF production and its receptor activity (20-24).

The effect of Celastrol on angiogenesis has been investigated in endothelial cells under normoxic conditions. However, intratumoral hypoxia is a common characteristic feature of solid tumors and it is the driving force for tumor angiogenesis and metastasis. Furthermore, in a complex tumor model, the effect of anticancer drugs not only on endothelial cells but also on tumor cells has to be taken into account. In the present study, we demonstrated that Celastrol could suppress hypoxia-induced angiogenesis and metastasis via blocking the migration as well as tube formation of endothelial cells and the invasion ability of cancer cells. Meanwhile, Celastrol directly inhibited the proliferation of endothelial and cancer cells under hypoxia. Our findings demonstrate the ability of Celastrol to inhibit angiogenesis and metastasis by targeting both tumor cells and surrounding endothelial cells under hypoxia, which exists in most solid tumors.

The critical mediator of the hypoxia-driven phenotypic changes is the transcription factor HIF-1, which acts directly or indirectly to promote the epidermal-mesenchymal transition, boost cancer invasiveness, increase production of angiogenic factors, and induce chemoresistance (19). In the current study, we have shown that Celastrol decreased the mRNA levels of HIF-1 $\alpha$ under both normoxic and hypoxic conditions. In addition, we found that Celastrol potently affected the nuclear translocation of HIF-1 $\alpha$ and reduced the hypoxia-induced nuclear accumulation of HIF- $1 \alpha$. Kallio et al have reported that the translocation of HIF-1 $\alpha$ from the cytoplasm to the nucleus is impaired in the presence of proteosome inhibitors (25). This might partly explain the effect of Celastrol on HIF-1 $\alpha$ translocation since Celastrol is a natural potent proteasome inhibitor (21). The result suggests that Celastrol possesses dual roles in HIF- $1 \alpha$ regulation, a direct action on its nuclear protein expression and a reduction of its mRNA levels. The dual activities are interesting because few HIF-1 $\alpha$ inhibitors, until now, possess the same properties.

The process of hypoxia-mediated angiogenesis is predominantly accomplished by an autocrine loop, acting through the HIF-1 $\alpha$ transcriptional regulation of the expression of VEGF and of other target genes. Our results have shown an increase of the mRNA and secreted protein levels of VEGF under hypoxia in accordance to other studies $(26,27)$. A decrease in VEGF mRNA and protein levels, which was associated with the inhibition of HIF-1 $\alpha$ expression by Celastrol was observed in Celastrol-treated hypoxic cells. To further investigate the effect of Celastrol on the transcriptional activity of HIF-1 $\alpha$, we examined the mRNA levels of CA9, which is a downstream target of HIF-1 $\alpha$. Celastrol dose-dependently inhibited hypoxia-induced expression of CA9 mRNA. The results suggest that Celastrol may affect HIF-1 $\alpha$ transcriptional activity and that reduction of VEGF secretion from the Celastrol-treated hypoxic tumor cells is attributable to the down-regulation of HIF-1 $\alpha$ mediated VEGF mRNA transcription.

Additionally, we have found that HIF- $1 \alpha$ protein degradation by Celastrol might partly be mediated via HSP90. HSP90 is an essential molecular chaperone that is involved in the post-translational folding and stability of a host of proteins that are important to the cancer cells, such as EGFR, CRAF, Akt, Met, HIF-1 $\alpha$, and telomerase (28). Zhang et al have reported that Celastrol disrupts the association of HSP90 with co-chaperones p23 and $\mathrm{Cdc} 37$ and allosterically regulates HSP90 chaperone activity by modifying its C-terminus, resulting in the degradation of HSP90 client proteins (29). Furthermore, we found that Celastrol did not cause a major change in HSP90 protein levels in agreement with previous observations (10). Elevated activation of Met and Akt, both HSP90 clients, has been detected in cancer cells, promoting cancer cell metastasis as well as survival. In our experiments, Celastrol treatment gradually down-regulated both Akt and Met as well as their phosphorylated forms in a dose-dependent manner. Thus, Celastrol may reduce HIF-1 $\alpha$ nuclear protein expression by suppressing HSP90 activity without changing the levels of HSP90 protein.

All of the known angiogenesis inhibitors significantly arrest tumor growth in preclinical studies, but, unfortunately, most of them fail in human trials. Due to the complexity of a tumor model, a single drug targeting a particular oncogene is unlikely to be entirely effective for cancer therapy. Thus, combinations of multiple drugs have been recommended for treatment of human cancer, because they may target various cellular pathways and would yield better outcomes than monotherapies. In this respect, Celastrol which contains various phytochemicals targeting multiple dysregulated pathways in cancer cells may provide an alternative/complementary way to treat cancer. In this study, we reveal a new role for Celastrol in inhibiting hypoxia-mediated angiogenesis and metastasis via regulating the HIF-1 $\alpha$ pathway. At least three activities of Celastrol may contribute to its antiangiogenic effect under hypoxia. Firstly, Celastrol directly inhibits the proliferation of hypoxic endothelial cells. Secondly, Celastrol impairs hypoxia-induced migration and tube formation of endothelial cells. Thirdly, Celastrol could down-regulate the mRNA and protein expression of the pro-angiogenic factor, VEGF, by hypoxic cancer cells. Two mechanisms may account for the Celastrol inhibition of VEGF in cancer cells. Firstly, we found that Celastrol could diminish the hypoxia-induced expression of HIF-1 $\alpha$, which is a transcriptional activator of the VEGF gene. Secondly, we demonstrated that Celastrol could decrease the expression of Akt and pAkt, which can increase VEGF expression via the HIF-1 $\alpha$ pathway.

The formation of distant metastasis is the main cause of morbidity and mortality in patients with cancer (30). Once a solid tumor spreads to other tissues and organs, curative intervention with currently available cancer drugs, surgical operation, and radiotherapy are ineffective. Hypoxia 
is likely to increase the capacity of cancer cells to invade healthy tissue, often co-opting the pre-existing vasculature of that tissue, and to form new metastases. In this study, we demonstrated that several activities of Celastrol may directly or indirectly account for its suppressive effect on cancer metastasis under hypoxia. Firstly, Celastrol dramatically inhibited the stimulatory effect of hypoxia on the invasion ability of cancer cells. Secondly, Celastrol could repress the expression of HIF-1 $\alpha$, which has been shown to regulate multiple steps in the complex process of metastasis. Thirdly, Celastrol could decrease the mRNA levels of CA9, which is overexpressed in a variety of tumor types and associated with increased metastases and poor prognosis (18). Fourthly, Celastrol could interrupt the activity of Met, a key molecule of the Met signaling pathway that has been shown to promote tumor invasion and metastasis.

To the best of our knowledge, this is the first documentation that Celastrol exerts its broad spectrum of antiangiogenic and antimetastatic effect through its potent inhibition of HIF-1 $\alpha$ and its downstream target genes, in the context of tumor hypoxia, a common feature of most solid cancers. These results provide new insight into the mechanisms of the antitumor action of Celastrol.

\section{Acknowledgements}

This study is primarily supported by grants from the Natural Science Foundation of China (no. 30973473). We thank Rui Meng, Ming Luo, Qing-Yao Zhu and Jin Su for technical assistance.

\section{References}

1. Hockel M and Vaupel P: Biological consequences of tumor hypoxia. Semin Oncol 28: 36-41, 2001.

2. Greco O, Marples B, Joiner MC and Scott SD: How to overcome (and exploit) tumor hypoxia for targeted gene therapy. J Cell Physiol 197: 312-325, 2003.

3. Brizel DM, Scully SP, Harrelson JM, Layfield LJ, Bean JM, Prosnitz LR and Dewhirst MW: Tumor oxygenation predicts for the likelihood of distant metastasis in human soft tissue sarcoma. Cancer Res 56: 941-943, 1996.

4. Semenza GL: Targeting HIF-1 for cancer therapy. Nat Rev Cancer 3: 721-732, 2003.

5. Wang GL and Semenza GL: Purification and characterization of hypoxia-inducible factor 1. J Biol Chem 270: 1230-1237, 1995.

6. Zhong H, De Marzo AM, Laughner E, Lim M, Hilton DA, Zagzag D, Buechler P, Isaacs WB, Semenza GL and Simons JW: Overexpression of hypoxia-inducible factor 1-alpha in common human cancers and their metastases. Cancer Res 59: 5830-5835, 1999.

7. Blagosklonny MV: Hypoxia-inducible factor: Achilles' heel of antiangiogenic cancer therapy. Int J Oncol 19: 257-262, 2001

8. Maxwell PH, Dachs GU, Gleadle JM, Nicholls LG, Harris AL, Stratford IJ, Hankinson O, Pugh CW and Ratcliffe PJ: Hypoxiainducible factor-1 modulates gene expression in solid tumors and influences both angiogenesis and tumor growth. Proc Natl Acad Sci USA 94: 8104-8109, 1997.

9. Salminen A, Lehtonen M,Paimela T and Kaarniranta K: Celastrol: molecular targets of Thunder God Vine. Biochem Biophys Res Commun 394: 439-442, 2010.

10. Zhang T, Hamza A, Cao X, Wang B, Yu S, Zhan CG and Sun D: A novel Hsp90 inhibitor to disrupt Hsp90/Cdc37 complex against pancreatic cancer cells. Mol Cancer Ther 7: 162-170, 2008.
11. Katschinski DM, Le L, Heinrich D, Wagner KF, Hofer T, Schindler SG and Wenger RH: Heat induction of the unphosphorylated form of hypoxia inducible factor- $1 \alpha$ is dependent on heat shock protein 90 activity. J Biol Chem 277: 9262-9267, 2002.

12. Isaacs JS, Jung YJ, Mimnaugh EG, Martinez A, Cuttitta F and Neckers LM: Hsp90 regulates a von Hippel Lindau-independent hypoxia inducible factor-1 alpha-degradative pathway. J Biol Chem 277: 29936-29944, 2002.

13. Zhou J, Schmid T, Frank R and Brune B: PI3K/Akt is required for heat shock proteins to protect hypoxia inducible factor $1 \alpha$ from pVHL-independent degradation. J Biol Chem 279: 13506-13513, 2004.

14. Ibrahim NO, Hahn T, Franke C, Stiehl DP, Wirthner R, Wenger RH and Katschinski DM: Induction of the hypoxiainducible factor system by low levels of heat shock protein 90 inhibitors. Cancer Res 65: 11094-11100, 2005.

15. Liao D, Corle C, Seagroves TN and Johnson RS: Hypoxiainducible factor-1alpha is a key regulator of metastasis in a transgenic model of cancer initiation and progression. Cancer Res 67: 563-572, 2007.

16. Boutin AT, Weidemann A, Fu Z, et al: Epidermal sensing of oxygen is essential for systemic hypoxic response. Cell 133: 223-234, 2008.

17. Potter C and Harris AL: Hypoxia inducible carbonic anhydrase IX, marker of tumour hypoxia, survival pathway and therapy target. Cell Cycle 3: 164-167, 2004.

18. Robertson N, Potter C and Harris AL: Role of carbonic anhydrase IX in human tumor cell growth, survival, and invasion. Cancer Res 64: 6160-6165, 2004.

19. McCarty MF, Barroso-Aranda J and Contreras F: Practical strategies for suppressing hypoxia-inducible factor activity in cancer therapy. Med Hypotheses 74: 789-797, 2010.

20. Sethi G, Ahn KS, Pandey MK and Aggarwal BB: Celastrol, a novel triterpene, potentiates TNF-induced apoptosis and suppresses invasion of tumor cells by inhibiting NF- $\kappa B$-regulated gene products and TAK1-mediated NF- $\mathrm{BB}$ activation. Blood 109: 2727-2735, 2007.

21. Yang H, Chen D, Cui QC, Yuan X and Dou QP: Celastrol, a triterpene extracted from the Chinese 'Thunder of God Vine,' is a potent proteasome inhibitor and suppresses human prostate cancer growth in nude mice. Cancer Res 66: 4758-4765, 2006.

22. Walcott SE and Heikkila JJ: Celastrol can inhibit proteasome activity and upregulate the expression of heat shock protein genes, hsp30 and hsp70, in Xenopus laevis A6 cells. Comp Biochem Physiol A Mol Integr Physiol 156: 285-293, 2010.

23. Pang X, Yi Z, Zhang J, Lu B, Sung B, Qu W, Aggarwal BB and Liu M: Celastrol suppresses angiogenesis-mediated tumor growth through inhibition of $\mathrm{AKT} / \mathrm{mammalian}$ target of rapamycin pathway. Cancer Res 70: 1951-1959, 2010.

24. Huang Y, Zhou Y, Fan Y and Zhou D: Celastrol inhibits the growth of human glioma xenografts in nude mice through suppressing VEGFR expression. Cancer Lett 264: 101-106, 2008.

25. Kallio PJ, Wilson WJ, O'Brien S, Makino Y and Poellinger L: Regulation of the hypoxia-inducible transcription factor 1 by the ubiquitin-proteasome pathway. J Biol Chem 274: 6519-6525, 1999.

26. Alqawi O, Moghaddas M and Singh G: Effects of geldanamycin on HIF- $1 \alpha$ mediated angiogenesis and invasion in prostate cancer cells. Prostate Cancer Prostatic Dis 9: 126-135, 2006.

27. van Lieshout T, Stanisz J, Espiritu V, Richardson M and Singh G: A hypoxic response induced in MatLyLu cells by cobalt chloride results in an enhanced angiogenic response by the chick chorioallantoic membrane. Int J Oncol 23: 745-750, 2003.

28. Banerji U: Heat shock protein 90 as a drug target: some like it hot. Clin Cancer Res 15: 9-14, 2009.

29. Zhang T, Li Y, Yu Y, Zou P, Jiang Y and Sun D: Characterization of celastrol to inhibit hsp90 and cdc37 interaction. J Biol Chem 284: 35381-35389, 2009.

30. Duffy MJ, McGowan PM and Gallagher WM: Cancer invasion and metastasis: changing views. J Pathol 214: 283-293, 2008. 